Résumés des conférences et travaux

\title{
Histoire et civilisation de l'écrit en Chine
}

Livres de voyages

Jean-Pierre Drège

\section{OpenEdition}

Journals

Édition électronique

URL : https://journals.openedition.org/ashp/334

DOI : 10.4000/ashp.334

ISSN : 1969-6310

Éditeur

Publications de l'École Pratique des Hautes Études

\section{Édition imprimée}

Date de publication : 1 octobre 2008

Pagination : 334-335

ISSN : 0766-0677

\section{Référence électronique}

Jean-Pierre Drège, "Histoire et civilisation de l'écrit en Chine », Annuaire de l'École pratique des hautes études (EPHE), Section des sciences historiques et philologiques [En ligne], 139 | 2008, mis en ligne le 07 janvier 2009, consulté le 12 juillet 2021. URL : http://journals.openedition.org/ashp/334 ; DOI : https:// doi.org/10.4000/ashp.334 


\title{
HISTOIRE ET CIVILISATION DE L’ÉCRIT EN CHINE
}

\author{
Directeur d'études : M. Jean-Pierre DrÈGE
}

Programme de l'année 2006-2007 : Livres de voyages.

Les conférences ont porté cette année sur des livres de voyage particuliers, à savoir des ouvrages puisant aux récits de voyage et s'arrêtant sur des populations de contrées extérieures aux coutumes particulières. Le caractère d'extériorité, de différence de certaines de ces populations, par rapport aux us et coutumes de la Chine à un moment donné, est souvent souligné par des caractéristiques qui expriment l'étrangeté de ces peuples. Cette étrangeté est également souvent proprotionnelle à l'éloignement des pays décrits, même si ce n'est pas toujours le cas.

Dans ce parcours, nous visions à approcher la perception des étrangers non dans un cadre historique, mais dans le contexte d'une littérature commune. Ont donc été convoqués d'abord le Huainanzi de Liu An (II siècle avant J.-C.), le Shanhai jing (III ${ }^{\mathrm{e}}$ et $\mathrm{II}^{\mathrm{e}}$ siècles avant J.-C.) et le Bowuzhi de Zhang Hua ( $\mathrm{III}^{\mathrm{e}}$ siècle de l'ère chrétienne), premiers témoignages d'une perception particulière des territoires étrangers. Puis, le Youyang zazu de Duan Chengshi (vers 800-863) dont les éléments concernés ont été intégrés dans l'encyclopédie littéraire du début des Song, le Taiping guangji. Les quatre chapitres du Taiping guangji portant sur les barbares des quatre orients ont été lus et commentés dans leur ensemble. Enfin une attention particulière a été donnée aux chapitres d'une autre encyclopédie beaucoup plus tardive, le Sancai tuhui de Wang Qi (fl. 1565-1614).

Le Shanhai jing, comme le Huainanzi, ne fournissent que des indications sommaires sur les peuples d'outre-mer. La lecture du Shanhai jing doit s'appuyer sur les commentaires, notamment ceux de Guo Pu (276-324) qui apportent une description minimale des êtres mentionnés. Le recours aux éditions illustrées du Shanhai jing, en particulier celle de Wang Chongqing (Shanhai jing shiyi), datée de 1537 a permis de mieux comprendre le fonctionnement de l'imagerie de ces peuplades étranges.

Avec le Taiping guangji (et le Youyang zazu), on a affaire à une autre approche. Les textes sont plus substantiels. Ils couvrent par ailleurs une étendue beaucoup plus grande, conforme aux connaissances plus larges d'une époque plus avancée. Ils s'attachent à présenter, souvent sous forme anecdotique, l'ensemble des populations étrangères connues par l'histoire et la géographie. On y retrouve les peuples étranges du Shanhai jing, mais également des peuples légendaires de l'Antiquité ainsi que des populations parfaitement connues et voisines de la Chine, comme les Turcs (Tujue), les Tibétains (Tufan) ou la Corée. Ici, c'est presque toujours dans un contexte merveilleux qu'ils apparaissent.

Le Sancai tuhui, pour sa part, présente un attrait particulier, puisqu'il s'agit d'une encyclopédie illustrée. Les brèves notes consacrées à chaque pays sont en effet accom- 
pagnées d'une image rappelant ou non les données du texte. Comme toutes les encyclopédies chinoises, il s'agit d'un recueil de citations mêlant des sources diverses, mais ici non mentionnées. On y retrouve des éléments du Youyang zazu, d'une autre encyclopédie datant de la dynastie des Song et enrichie sous les Yuan, le Shilin guangji de Chen Yuanqing (vers 1200-1266), d'ouvrages consacrés totalement ou partiellement aux pays étrangers, comme le Lingwai daida de Zhou Qufei (mort après 1178) ou le Yiyu zhi de Zhou Zhizhong (XIV siècle). Les pays étrangers figurent dans la section des Hommes du Sancai tuhui, qu'ils terminent. Dans le chapitre 12, chaque pays occupe deux pages, l'une pour le texte, l'autre pour l'image. Dans les chapitres 13 et 14, chaque pays n'occupe qu'une page où se répartissent texte et image. En tout ce sont 175 pays ou peuplades qui défilent, depuis les royaumes de Sinluo et Koryo, c'està-dire de Corée, jusqu'à La Mecque, dont on fait le lieu de naissance de la divinité locale Maxia (Mahomet) que l'on appelle là-bas « bouddha ». Si le premier des trois chapitres contient des notes entièrement consacrées à des pays réels, les deux autres mêlent les données de livres de voyages des Song et des Yuan avec les vieilles descriptions du Shanhai jing. Le plus souvent chaque pays est caractérisé par des éléments significatifs de la vie locale qui le distinguent des autres. Ceux-ci sont souvent assez surprenants, comme par exemple les pratiques sexuelles du Xian (Siam), les coutumes matrimoniales du Zhenla (Cambodge), avec leurs curieux rites de défloration, ou de la côte somalienne (Dashi Bibaluo). On s'est arrêté assez longuement sur les coutumes macabres des « têtes volantes » ou des barbares « têtes de cadavre » qui appartiennent tantôt aux barbares Liao de la Chine du Sud, tantôt au royaume du Panduranga ou du Champa, où se trouvent des femmes sans pupille dont la tête s'en va errer la nuit. Cette sorte de vampire ne peut être supprimée qu'en empêchant la tête de rejoindre le corps au petit matin. Le dernier chapitre du Sancai tuhui traitant des étrangers s'achève carrément par un inventaire de chimères et d'êtres fantastiques circulant dans les montagnes comme ceux que révéle le Shanhai jing.

Le caractère cumulatif des encyclopédies chinoises a fréquemment pour conséquence, jusqu'à une période tardive, l'absence d'une perspective rationnelle. Le savoir exposé mêle sans distinction le réel et l'imaginaire, quelle que soit l'époque. C'est le cas dans le Sancai tuhui, c'est encore le cas plus tard, à la fin du XVIII ${ }^{\mathrm{e}}$ siècle, avec la grande encyclopédie du Tushu jicheng qui reprend aux encyclopédies précédentes et accroît le nombre des notes sur les pays étrangers en conservant cette ambiguité. 